\title{
World Lines in Einstein's Elevator
}

\author{
Mathieu Rouaud* \\ Boudiguen 29310 Querrien, France \\ * mathieu137@gmail.com
}

May 31, 2021

\begin{abstract}
We all have in mind Einstein's famous thought experiment in the elevator where we observe the free fall of a body, and then the trajectory of a light ray. Simply here, in addition to the qualitative aspect, we carry out the exact calculation. We consider a uniformly accelerated reference frame in rectilinear translation, and we show that the trajectories of the particles are ellipses centered on the event horizon. The frame of reference is non-inertial, the space-time is flat, the metric is non-Minkowskian, and the computations are performed within the framework of special relativity. Some experimental consequences are discussed, such as the deviation of trajectories, the desynchronization of a falling clock, the accelerated Michelson-Morley experiment, and, finally, an experiment where a paradox appears - a particle of matter seems to go faster than light. The differences, compared to the classical case, are important at large scale and close to the horizon, but they are small in the lift where the interest is above all theoretical and pedagogical. The study helps the student to become familiar with the concepts of metric, coordinate velocity, horizon, and, to do the analogy with the black hole.
\end{abstract}

Keywords: uniformly accelerating, worldline, circle, synchronization, Michelson, interferometer, paradox.

\section{Contents}

\begin{tabular}{lll}
\hline 1 & Introduction & 2
\end{tabular}

\begin{tabular}{|lll}
2 & Uniformly accelerated reference frame & 3
\end{tabular}

2.1 Coordinate system 3

\begin{tabular}{|ll}
\hline 2.2 & Metric and change of coordinates
\end{tabular}

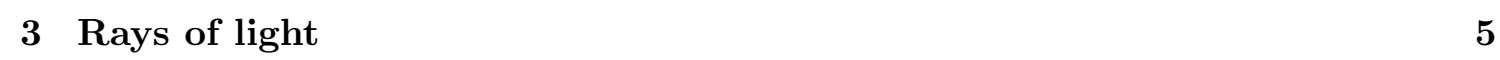

\begin{tabular}{ll|l}
3.1 & Lateral rays & 5
\end{tabular}

\begin{tabular}{lll}
\hline 3.2 & Any rays & 6
\end{tabular}

\begin{tabular}{lll}
\hline 3.3 & Horizontal synchronization & 8 \\
\hline 3.4 & Radar & 8
\end{tabular}

$\begin{array}{lll}3.4 & \text { Radar distance } & 8\end{array}$

\begin{tabular}{|lll}
4 & Non-zero mass particles & 9
\end{tabular}

$\begin{array}{lll}4.1 & \text { Trajectories and worldlines } & 9\end{array}$

\begin{tabular}{|lll}
\hline 4.2 & Velocity and acceleration for a particle released at rest & 10
\end{tabular}

\begin{tabular}{lll}
\hline 4.3 & Comparison with the black hole & 11
\end{tabular} 
$\begin{array}{lll}5 & \text { Experimental aspects } & \mathbf{1 3}\end{array}$

$\begin{array}{lll}5.1 & \text { Deviation measurement } & 13\end{array}$

$\begin{array}{lll}5.2 & \text { Desynchronization of clocks } & 13\end{array}$

\begin{tabular}{lll}
\hline 5.3 & Michelson interferometer & 13
\end{tabular}

$\begin{array}{lll}5.4 & \text { Three rockets experiment } & 15\end{array}$

$\begin{array}{lll}6 & \text { Conclusion } & 16\end{array}$

$\begin{array}{lr}\text { References } & 16\end{array}$

\section{Introduction}

We imagine a portion of empty space infinitely distant from all masses. We have a large box in which an observer floats in weightlessness. With the help of a hook and a rope, a constant force is exerted on the box, thus animated by a rectilinear translation motion uniformly accelerated. The observer then experiences an artificial gravity. We will study in the reference frame of the elevator the motion of light, then of a massive particle, and, finally, we will make a comparison with the black hole during a free fall from rest.

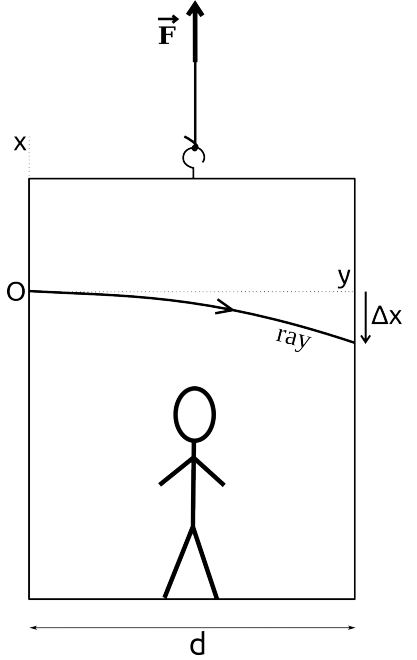

Figure 1:

Einstein's Elevator.

In the frame of the box, first inertial, a light beam propagates at speed $c$ along a straight line trajectory. Then, the box is accelerated and a ray, initially perpendicular to the direction of motion, follows a curved trajectory. Let us quote Albert Einstein in his book Relativity [1]: "It can easily be shown that the path of the same ray of light is no longer a straight line".

Following the birth of special relativity in 1905, the accelerated elevator thought experiment, proposed by Einstein as early as 1908, allowed to draw the analogy with gravity, to develop an intuition and to guide the foundation of general relativity. New theory of gravitation established in 1915 which then supplanted Newton's theory. This image was used to illustrate the principle of equivalence, and to predict the deviation of light rays by a massive star.

Nevertheless, the historical development of the theory should not derail attention from an fundamental point: the deviation of a light ray in the accelerated elevator is fully explained with the special theory of relativity. Indeed, in the accelerating reference frame of the elevator, spacetime remains flat ; no gravitational field here, and the deviation of light rays is understood by a purely kinematic reasoning.

According to Einstein's second postulate, the speed of light in vacuum has the same value $c$ in all inertial frames of reference. A logical consequence of this postulate: in a non-inertial frame the speed of light can, a priori, be different than $c$. . Another property: a free particle follows a rectilinear and uniform trajectory in an inertial frame. Therefore, a free particle can follow a curved trajectory in a non-inertial frame. As we will show, this is precisely what happens here for the ray of light.

\footnotetext{
${ }^{1}$ It remains, of course, that an object can in no case exceed the speed of light in vacuum, and that the speed of light for a local Minkowskian observer is always equal to $c$.
} 


\section{Uniformly accelerated reference frame}

\subsection{Coordinate system}

We want to describe physics from the point of view of the accelerated observer. A reference frame is a physical entity to which we associate a coordinate system for localization. A uniformly accelerated reference frame $R$ is defined as a set of observers who remain at rest with respect to each other in a hypothetical rigid three-dimensional structure. We imagine a continuous set of infinitesimal observers each equipped with a ruler and a clock [2] 3]. An event is uniquely specified with the observer on whose world-line the event occurs. We decide to assign a set of three numbers $(x, y, z)$ to each observer, and a number $t$ to each point of their worldline. For an inertial frame $R^{\prime}$, all the clocks can be synchronized, and $t$ corresponds to the proper time of each observer at rest in $R^{\prime}$. For a non-inertial frame, this is no longer possible and each observer has a second clock called coordinate clock. However, the uniformly accelerated frame is still rigid, because the relationships between the observers remain unchanged. We choose an observer $O$ of $R$ used as reference: $x=0$, and in his particular case the proper and coordinate times remain equal. By shifting the ruler as much as necessary in the directions of the proper acceleration given by an accelerometer, we assign an $x$ to each observer $X$. We proceed in the same way in two orthogonal directions for $y$ and $z$. $O$ emits a periodic light signal, and each of the other observers $X$ equals the rate of its coordinate clock with the signal received from $O$. To synchronize all the coordinate clocks, we use the radar method: $O$ sends a signal at $t_{1}$ reflected by $X$, and back to $O$ at $t_{2}$. When the signal is reflected by $X$, the time $t=\left(t_{1}+t_{2}\right) / 2$ is assigned to its coordinate clock. A coordinate clock can be compared to a clock radio-controlled by $O$ 's proper clock.

The coordinate system has been built intrinsically with the non-inertial observers. To define a constant acceleration for $O$ in an inertial frame $R^{\prime}$, we consider $R^{\prime}$ coinciding instantaneously with $R$, and then it is easy to demonstrate that the proper acceleration $\vec{a}_{p}$ is equal to the acceleration $\vec{a}$ of $R^{\prime}$ with respect to $R$. At this instant $t^{\prime}$, all clocks of $R^{\prime}$ and coinciding are set to zero. If later at $t$, a second inertial frame $R^{\prime \prime}$ coincides with $R$, the set of coordinate clocks of $R$ will still be synchronized, and we will be able to equalize their dates with a set of synchronized proper clocks of $R^{\prime \prime}$, however the set of proper clocks of $R$ will no longer be synchronized especially as $x$ is large.

A reference system where the clocks can be synchronized over all space is called a synchronous reference system [4]. A reference frame described by a metric $g_{\mu \nu}$ where the components, with one temporal index and the other spatial, are zero, is synchronous. As we will see in the following section 2.2 , this is the case of the uniformly accelerated reference frame: $g_{0 i}=0$ with $\mathrm{i}=1,2$ or 3 .

We can also synchronize clocks located in the same horizontal plane of $R$ (protocol described in section 3.3). There is invariance under translation along $y$ and $z$, and, in this case, the proper clocks are sufficient because the rhythm of the clocks is the same for a given $x$.

When a particle passes close to an observer, to measure its velocity and acceleration, he can use one or the other of his clocks: coordinate velocity and acceleration, $v^{\mu}=d x^{\mu} / d t$ and $a^{\mu}=d v^{\mu} / d t$, or local, $d x^{\mu} / d \tau$ and $d^{2} x^{\mu} / d \tau^{2}$. In an inertial reference frame, the speed of light is constant: distances can be measured equally with a ruler or a radar. In a noninertial frame, the coordinate velocity of light varies: distances measured by each method are generally different [5]. 


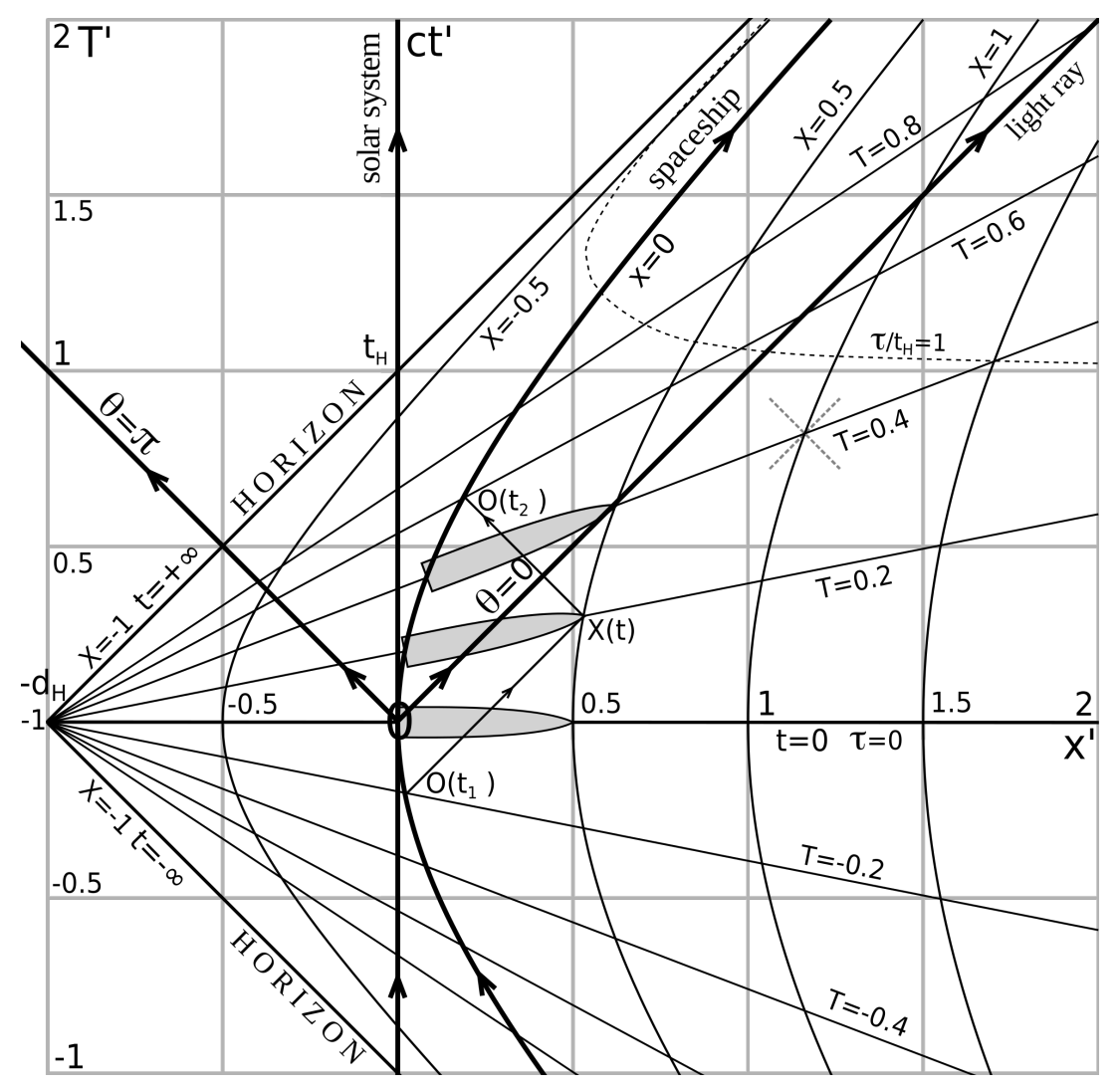

Figure 2: Minkowski diagram in $R^{\prime}$ inertial. The uniformly accelerated rocket has a hyperbolic worldline. We have represented the coordinate lines of $R^{\prime}$ and $R$ in $R^{\prime}$. In both cases, the coordinate lines of space and time are orthogonal. In this case, the rocket defines a rigid body of reference [2] 6] [3] with respect to which we can study the trajectory of the light rays.

\subsection{Metric and change of coordinates}

In a non-inertial frame of reference, the metric is non-Minkowskian. We consider the frame $R$ in rectilinear translation and uniformly accelerated with respect to $R^{\prime}$ inertial. We have the coordinate system $(c t, x, y, z)$ and we give the metric for a particle and a constant acceleration $\vec{a}=a \vec{u}_{x}[6]$ [7]:

$$
d s^{2}=c^{2} d \tau^{2}=g_{\mu \nu} d x^{\mu} d x^{\nu}=\left(1+\frac{a x}{c^{2}}\right)^{2} c^{2} d t^{2}-d x^{2}-d y^{2}-d z^{2} .
$$

The calculation shows that all the components of the Riemann curvature tensor are zero 8$]$, so the spacetime is flat, and there is a global change of coordinates from $R$ to $R^{\prime}[6]$ :

$$
c t^{\prime}=\left(x+\frac{c^{2}}{a}\right) \operatorname{sh}\left(\frac{a t}{c}\right), \quad x^{\prime}=\left(x+\frac{c^{2}}{a}\right) \operatorname{ch}\left(\frac{a t}{c}\right)-\frac{c^{2}}{a}, \quad y^{\prime}=y, \quad z^{\prime}=z .
$$

with $\vec{u}_{x^{\prime}}=\vec{u}_{x}, x^{\prime}\left(t^{\prime}=0\right)=x(t=0)=0$ and $v_{x^{\prime}}^{\prime}\left(t^{\prime}=0\right)=0$. In $R^{\prime}$, we find back the Minkowski metric: $d s^{2}=d s^{\prime 2}=c^{2} d t^{\prime 2}-d x^{\prime 2}-d y^{\prime 2}-d z^{\prime 2}$.

Let's use dimensionless quantities to simplify the study. For distances, $X=x / d_{H}$ with the horizon distance $d_{H}=c^{2} / a$. For times, $T=t / t_{H}$ with the horizon time $t_{H}=c / a$. For an acceleration equivalent to the intensity of gravity at the earth's surface, the horizon quantities are approximately one light-year and one year. To further illustrate our point, we can associate the reference frame $R^{\prime}$ to the galactic reference frame where stars are 
supposed to be fixed, and replace the elevator frame $R$ by the frame of a rocket that makes an interstellar voyage. The hyperplane $x=-d_{H}$ defines the event horizon as a break in the causal link. The astronauts will see their starting point, the Earth, moving away, then stopping at a light-year with a proper time frozen at one year.

The proper acceleration felt by an observer is inversely proportional to the horizon distance: $a_{p}=a /(1+X)$, so the rigidity is ensured.

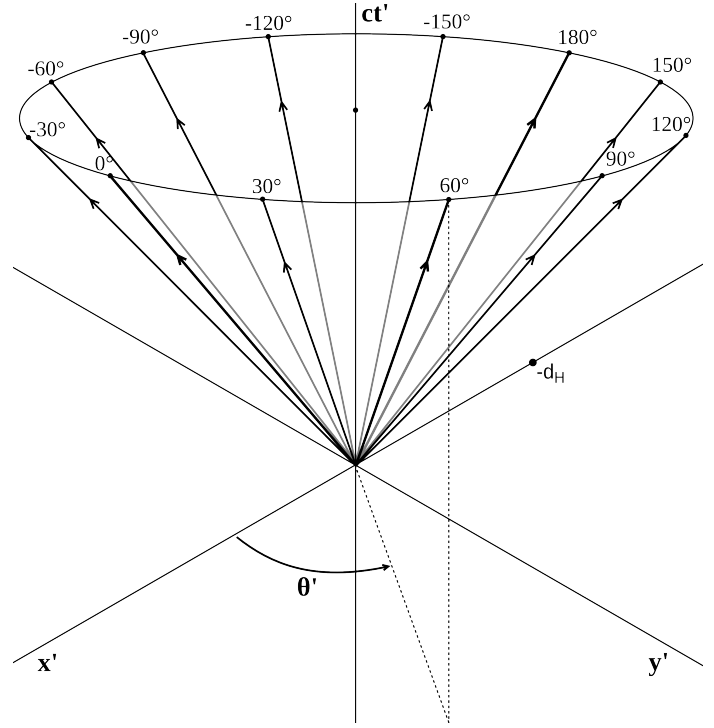

Figure 3: The worldlines of 12 particular rays in a Minkowski diagram $\left(T^{\prime}, X^{\prime}, Y^{\prime}\right)$ with $\theta^{\prime}\left(t^{\prime}=0\right)=\theta(t=0)$ as initial conditions.

\section{Rays of light}

Without losing in generality, we take as initial condition a light ray that enters the elevator when it has a zero speed in the inertial reference frame. All clocks are then set to zero, and the angle of entry of the ray is the same for all observers. The trajectories are all located in the plane $z^{\prime}=z=0$.

\subsection{Lateral rays}

In $R^{\prime}$, the ray worldline equations are $Y^{\prime}=T^{\prime}$ and $X^{\prime}=0$. Also $Y^{\prime}=Y$, hence we have [6] [8]:

$$
X=1 / \operatorname{ch} T-1, \quad Y=t h T \quad \Rightarrow \quad(X+1)^{2}+Y^{2}=1 .
$$

The ray of light traces a quarter circle of radius $d_{H}$ and center $\left(-d_{H}, 0\right)$. For a light ray $d \tau=0$ then $|\beta|=1+X$ with $\beta=d L / d T .|\beta| \neq 1$ because the coordinate system $(x, t)$ uses different physical methods of measuring distance and time: rods for $x$ and $O$ 's clock for $t$. The coordinate velocity of the light ray, initially equal to $c$, decreases and becomes zero on the horizon in an infinite time. As the components of the metric tensor depend on the point, the coordinate velocity corresponds to the local velocity just for the particular observer $O$, whereas for an inertial frame this velocity keeps the same meaning for all observers. For example, for the uniformly accelerated frame, the notion of rigidity is maintained, but, on the other hand, we can no longer define a set of proper clocks synchronized on the worldline of a particle. For the observer $O$, the measurements of his observers placed at different $x$ 's with their coordinate clock tell him that everything goes slower downwards, and faster upwards. This is why, from his point of view, light goes 


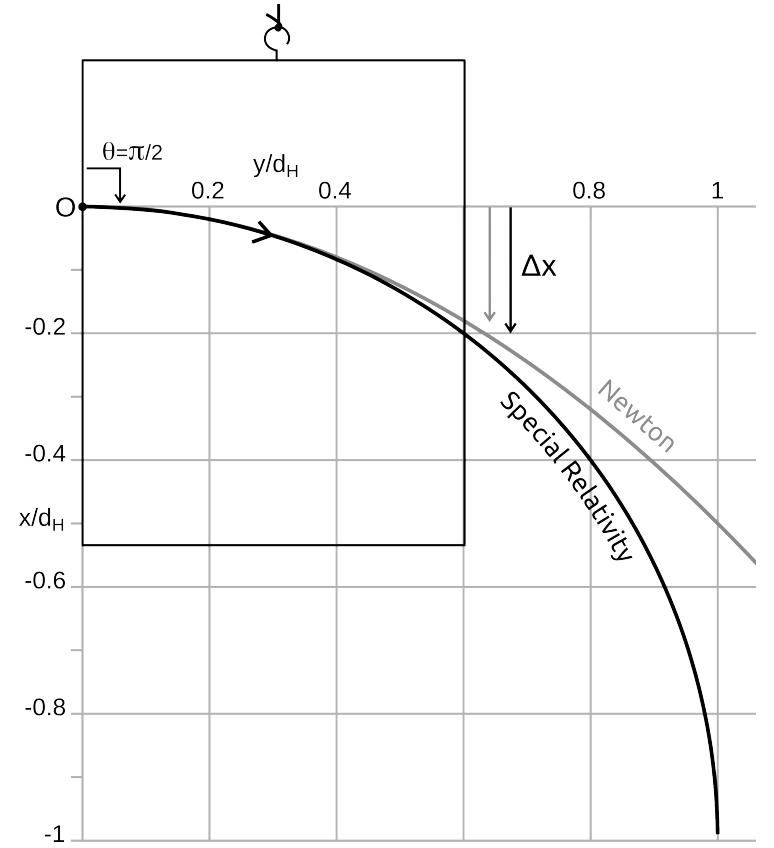

Figure 4: The circular trajectory of the ray traced on a vertical wall of the elevator. The deviation is greater than that of Newton's parabolic trajectory. At the level of the horizon the deviation is double.

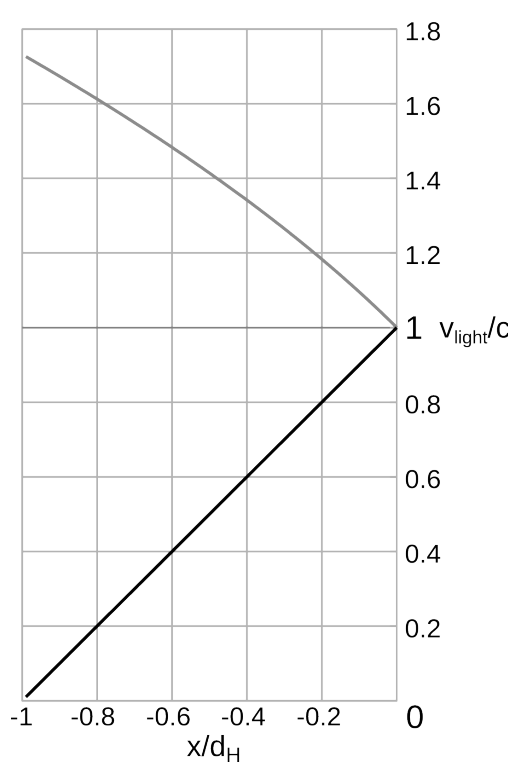

Figure 5: The speed of light which decreases and tends towards zero in $x=-d_{H}$. In classical theory, there is no horizon and the speed of light increases.

slower at lower levels, while a lower observer measures, where he is with his proper clock, a speed of light well equal to $c$.

For comparison, we give the predictions of Newton's theory: $X=-Y^{2} / 2$ and $\beta=$ $\sqrt{1-2 X}$. In this approximation, the trajectory is parabolic and the speed of light increases towards infinity.

\subsection{Any rays}

In general, the worldline of a ray in $R^{\prime}$ has for equations $Y^{\prime}=T^{\prime} \sin \theta^{\prime}$ and $X^{\prime}=T^{\prime} \cos \theta^{\prime}$. The calculation gives $(X+1)^{2}+(Y-1 / \tan \theta)^{2}=1 / \sin ^{2} \theta$. The ray of light describes a circular trajectory of radius $d_{H} /|\sin \theta|$ and center $\left(-d_{H}, d_{H} / \tan \theta\right)$. The speed of a luminous ray, no matter the initial conditions of the trajectory, varies linearly with $X$. If $X>0$, the ray goes faster than $c$; if $X<0$, the speed of light becomes less than $c$, and tends towards zero when $X$ tends towards -1 .

Let us imagine that we place a large drawing sheet on the vertical wall $(O x y)$ of the elevator and that we reproduce with a pen the passage of the light rays on the sheet. We then detach the drawing, and, back in an inertial reference frame, present it to geometricians who will attest that they are indeed arcs of circles.

For vertical rays, we give the speed and the equations of the worldlines:

$$
\beta=d X / d T= \pm(1+X) \quad \text { then } \quad T= \pm \ln (1+X), \quad Y=0 .
$$

Newton's parabolic trajectory: $X=-Y^{2} /\left(2 \sin ^{2} \theta\right)+Y / \tan \theta$. For example, if the light ray initially goes forward, its speed will first decrease, and then the ray will return downward with its speed increasing and tending towards infinity. A notable difference with the classical theory is that the relativistic rocket cannot overtake the ray sent forward. 


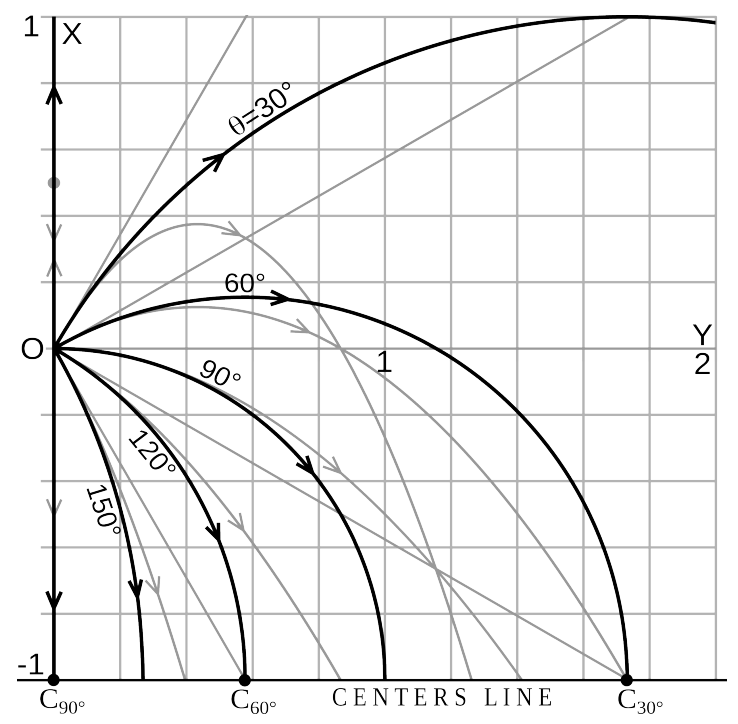

Figure 6: In the $(X, Y)$ plane, the trajectories of non-vertical light rays are portions of circles centered on the horizon. The rays thus arrive perpendicularly to the line of centers which is identified with the horizon. The grayed straight lines correspond to the inertial trajectories, in case the rocket is not accelerated. The gray parabolic lines are the Newtonian trajectories.

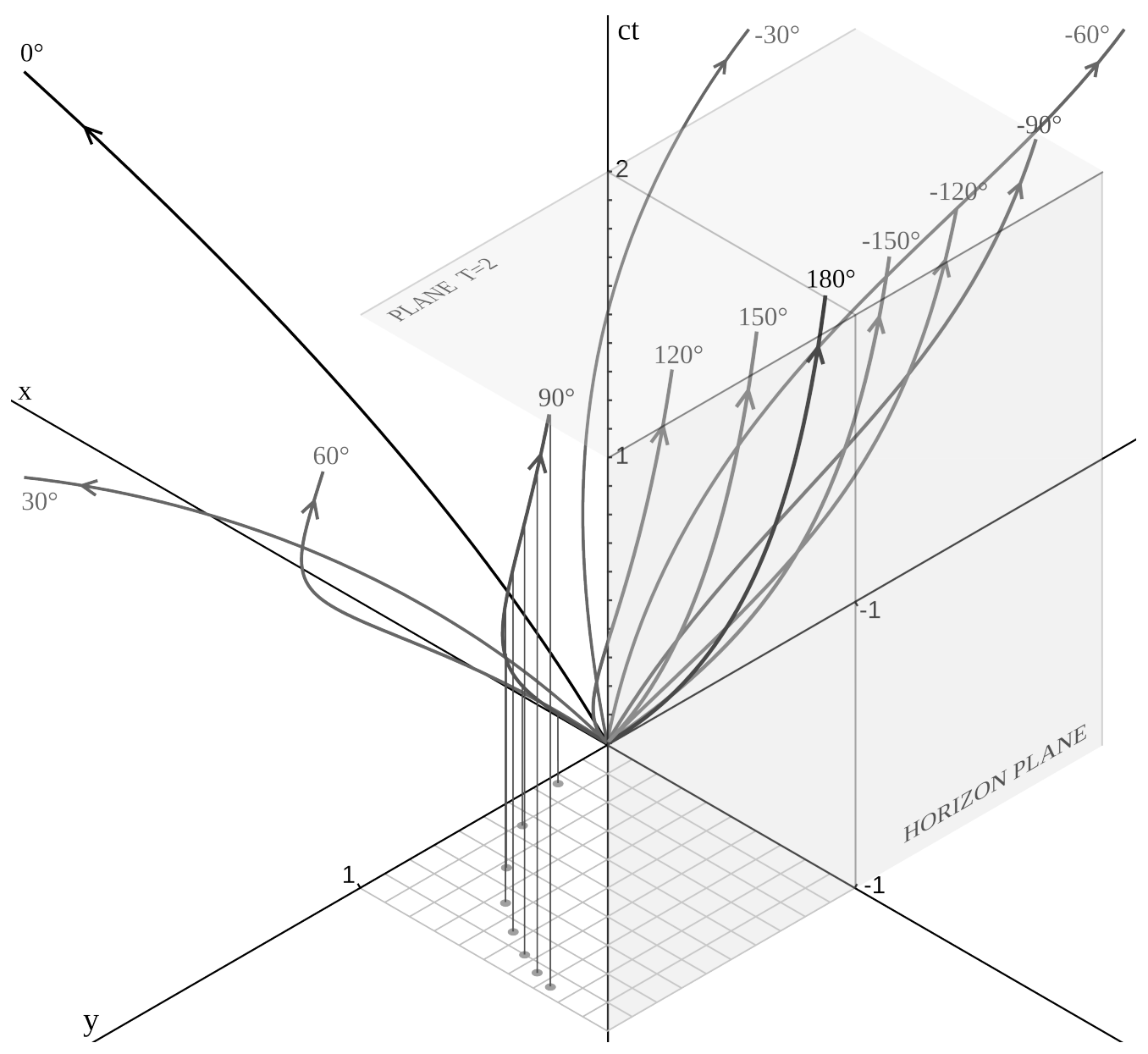

Figure 7: The Minkowski diagram in the non-inertial reference frame $(T, X, Y)$. Apart from the limit case $\theta=0$, all the light rays follow a partially helical worldline that asymptotically joins the horizon plane $x=-d_{H}$ in an infinite time $t$. The maximum deviation tends towards the half-turn for a ray emitted in the direction close to $\theta=0^{\circ}$. 


\subsection{Horizontal synchronization}

To synchronize clocks, we can use coinciding inertial reference frames, or, the radar method directly from the non-inertial reference frame. In an inertial reference frame, the light rays are straight and the speed of light is constant. In the Einstein elevator, the trajectories are circular and the speed of light varies. Nevertheless, the method is analogous and the round-trip time allows to synchronize the clock $B$ with the one in $A$. There is an angle $\theta$ for which the ray returns on its steps.

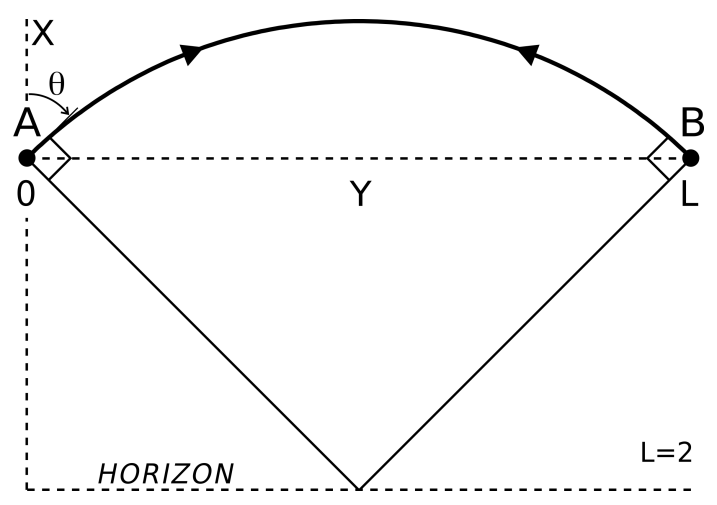

Figure 8: Two clocks $A$ and $B$ at the same level are synchronized with the radar method. A light beam is emitted by $A$, reflected in $B$ by a catadioptric system, and, back in $A$ after $2 \Delta t_{A B}$.

\subsection{Radar distance}

In an inertial reference frame, to measure distances, it was equivalent to use a ruler or the radar method. In the rocket, spatial geometry is Euclidean [5] and the use of rulers is natural ; on the other hand, the radar method must be adapted. For a pair of clocks $A$ and $B$ in a vertical plane, there is a unique semi-circle centered on the horizon that passes through $A$ and $B$, and thus a unique pair $(T=\Delta T, \theta)$ that allows to determine the position of $B$ with respect to $A$ :

$$
X=\frac{1}{\operatorname{ch} T-\operatorname{sh} T \cos \theta}-1, \quad Y=\frac{\operatorname{sh} T \sin \theta}{\operatorname{ch} T-\operatorname{sh} T \cos \theta} \quad \text { and } \quad L=\sqrt{X^{2}+Y^{2}} .
$$

For two clocks at the same level, the time interval $2 \Delta t$ of the radar echo is no longer proportional to the distance $l$, but, we can nevertheless express $l$ as a function of $\Delta t$ :

$$
X=0 \quad \Rightarrow \quad L=\sqrt{2(\operatorname{chT}-1)} \quad \text { and } \quad \sin \theta=1 / \sqrt{1+L^{2} / 4} .
$$

In the elevator, for $l=10 \mathrm{~m}$ and $a=10 \mathrm{~m} / \mathrm{s}^{2}$, we can continue to consider straight rays with velocity $c$, indeed

$$
\delta \Delta T=\Delta T_{\text {inertial }}-\Delta T_{\text {non in. }}=L-a c h\left(1+\frac{L^{2}}{2}\right) \simeq \frac{L^{3}}{24} \quad \text { and } \quad \delta \Delta t \simeq \frac{l^{3} a^{2}}{24 c^{5}}
$$

then $\delta \Delta t \simeq 1.7 \times 10^{-40} s$, well beyond the accuracy of the best atomic clocks. To increase the distance on the scale of the solar system, we can also exchange light rays between two rockets $A$ and $B$ whose parallel accelerations are synchronized. Over a distance of one astronomical unit $\delta \Delta t \simeq 5.8 n s$ for $\Delta t \simeq 8 \mathrm{~m} 20 \mathrm{~s}$. 


\section{Non-zero mass particles}

\subsection{Trajectories and worldlines}

The worldline of a particle in $R^{\prime}$ has for equations $Y^{\prime}=\beta_{0} T^{\prime} \sin \theta^{\prime}$ and $X^{\prime}=\beta_{0} T^{\prime} \cos \theta^{\prime}$ with $\beta_{0} c$ the initial velocity. The calculation gives $(X+1)^{2} / R_{X}{ }^{2}+\left(Y-Y_{C}\right)^{2} / R_{Y}{ }^{2}=1$ with $R_{X}=a / d_{H}=1 / \sqrt{1-\beta_{0}{ }^{2} \cos ^{2} \theta}$ and $R_{Y}=b / d_{H}=\beta_{0}|\sin \theta| R_{X}{ }^{2}$. The particle describes an elliptical trajectory of semi-major axis $a$, of semi-minor axis $b$ and center $\left(-d_{H}, \beta_{0}^{2} \sin \theta \cos \theta R_{X}^{2} d_{H}\right)$.

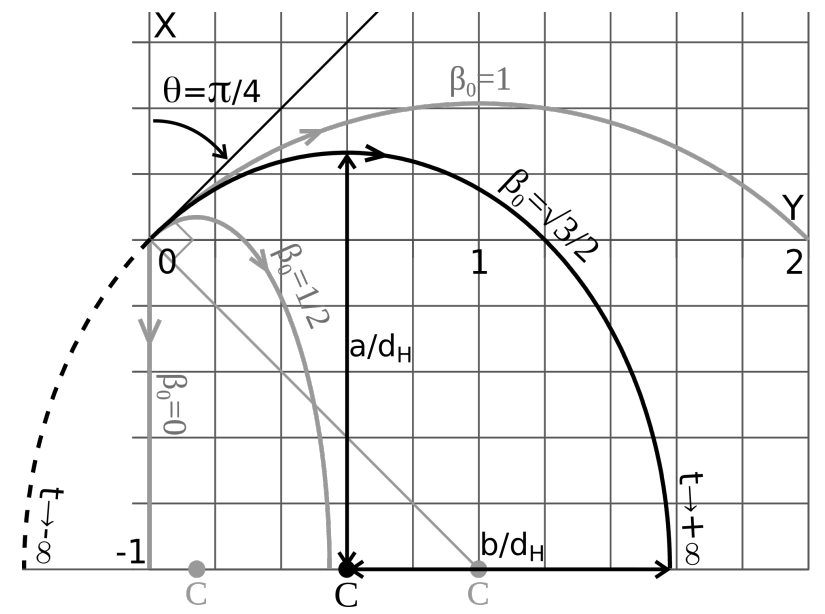

Figure 9: In the $(X, Y)$ plane, the trajectories of the particles are portions of ellipses centered on the horizon. The trajectories are perpendicular to the horizon line. The trajectory from $t \rightarrow$ $-\infty$ to $t \rightarrow+\infty$ is a semi-ellipse, or a semi-circle for a zero-mass particle.

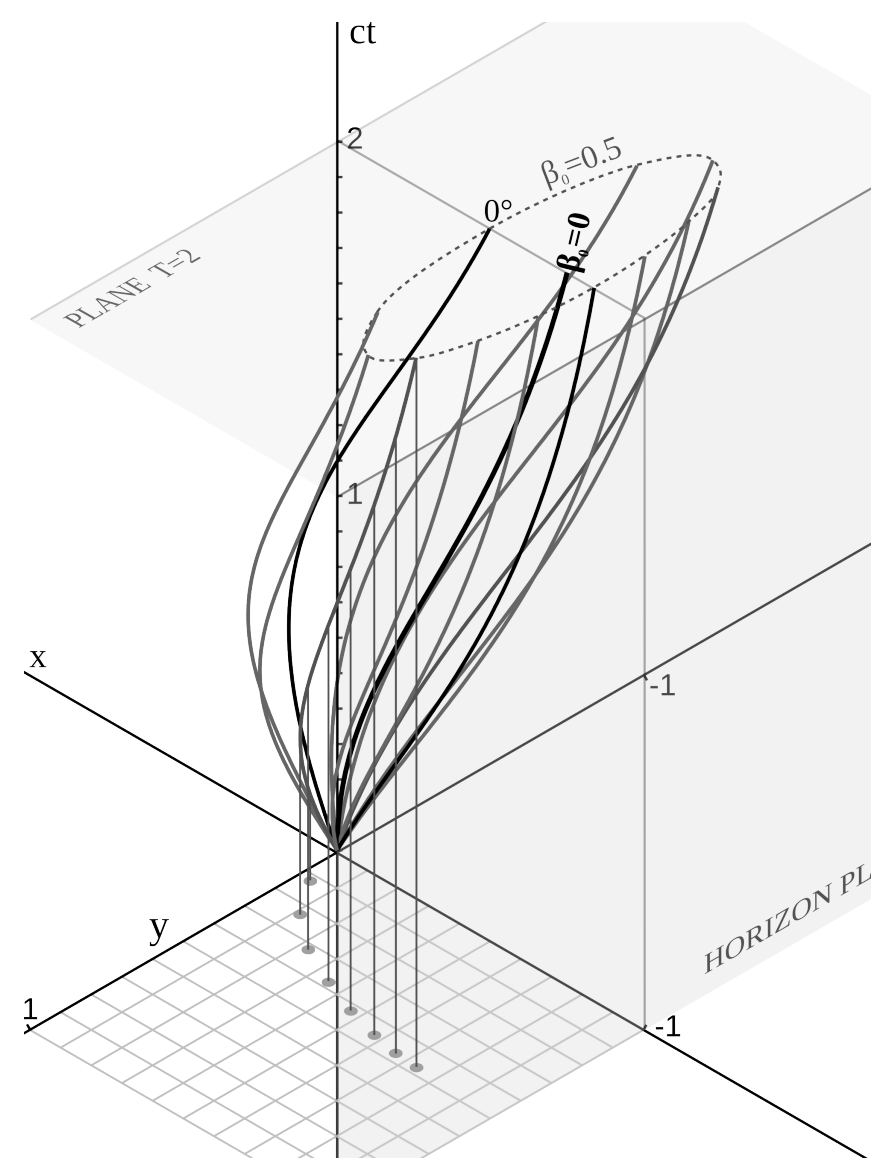

Figure 10: The Minkowski diagram in the non-inertial reference frame $(T, X, Y)$. Worldlines for $v_{0}=50 \%$ of $c$. In bold, the worldline for a particle released at rest.

The intersection between a horizontal plane and the tubular worldsheet, formed by the set of worldlines for different $\theta$ 's and a given $\beta_{0}$, also forms an ellipse (dotted curve). 
World lines equations:

$$
X=\frac{1}{c h T-\beta_{0} s h T \cos \theta}-1 \quad \text { and } \quad Y=\frac{\beta_{0} s h T \sin \theta}{c h T-\beta_{0} s h T \cos \theta} .
$$

Newton's parabolic trajectory: $X=-Y^{2} /\left(2 \beta_{0}^{2} \sin ^{2} \theta\right)+Y / \tan \theta$.

\subsection{Velocity and acceleration for a particle released at rest}

The initial speed of the particle is zero and the motion is vertical:

$$
\begin{aligned}
X=\frac{1}{c h T}-1, \quad \beta=\frac{d X}{d T}=-\frac{t h T}{c h T}= & -\sqrt{1-(X+1)^{2}}(X+1), \\
A & =\frac{d \beta}{d T}=\frac{c h^{2} T-2}{c h^{3} T}=\left[1-2(X+1)^{2}\right](X+1)
\end{aligned}
$$

We have $v_{\max }=c / 2$ at $X\left(v_{\max }\right)=1 / \sqrt{2}-1 \simeq-0.3$ [9] [3]. Initially the speed increases, then it decreases and tends to zero on the horizon. The acceleration is first negative, then zero and becomes positive. The elevator observer can initially interpret the metric effects as an inertial force similar to gravitation, but then his measurements will lead him to interpret a repulsive force as the particle approaches the horizon. These are spatiotemporal perspective effects experienced by the observer of the rocket. These metric effects due to the non-inertial character of the frame of reference are sometimes described in terms of fictitious forces, because the particle in $R^{\prime}$ inertial does not undergo any force and follows a rectilinear and uniform trajectory.

For Minkowskian observers, instantaneously at rest in successive inertial reference frames that coincide at every moment with $R: d t_{\text {Mink }}=(1+X) d t$ then $v_{\text {Mink }}\left(x_{v_{\max }}\right)=$ $c / \sqrt{2} \simeq 71 \% c$ also $v_{\max }=v_{\text {light }} / \sqrt{2}$.

For the proper time, the metric gives $\tau(X)=t_{H} \sqrt{1-(1+X)^{2}}$. We have a quarter circle in the plane $\left(\tau / t_{H}, X\right)$. For example, as shown in figure 13 , when the falling speed is maximum, $T=a \operatorname{ch} \sqrt{2} \simeq 0.9$ and $\tau / t_{H}=1 / \sqrt{2} \simeq 0.7$. And for the observer of the vessel at $O$, the time of the object in free fall freezes, and tends towards $t_{H}$ when the time of the clock at $O$ tends towards infinity. When a free-falling observer crosses the horizon, apart from the breaking of the causal link with the vessel, nothing special happens and his proper time continues to elapse indefinitely.

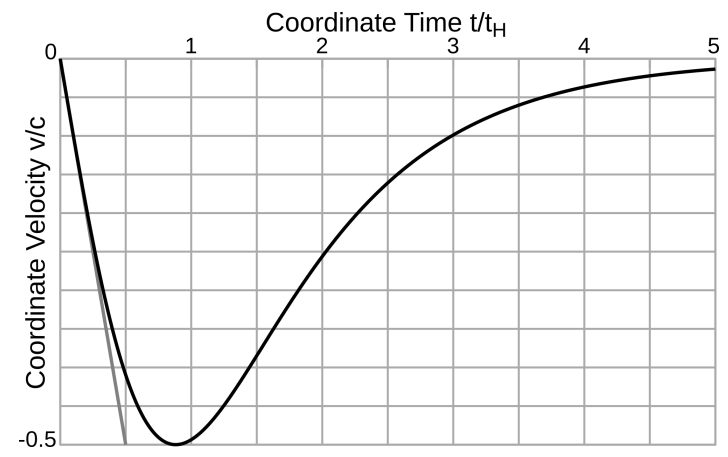

Figure 11: Falling velocity of a particle released at rest from $O$ at $T=0$. In gray, the classical case.

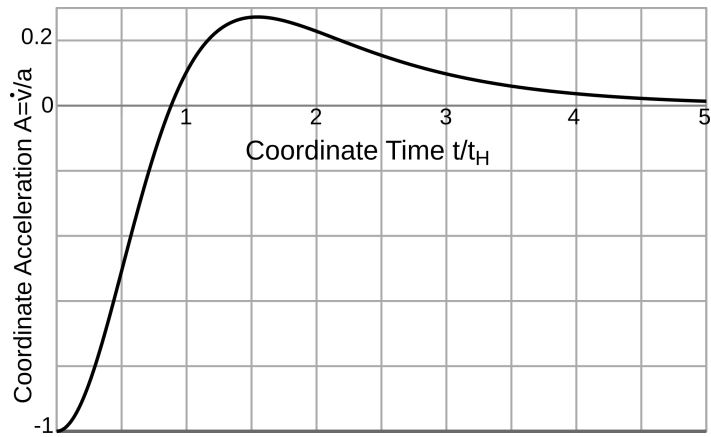

Figure 12: Particle acceleration during a free fall in the uniformly accelerating reference frame. 


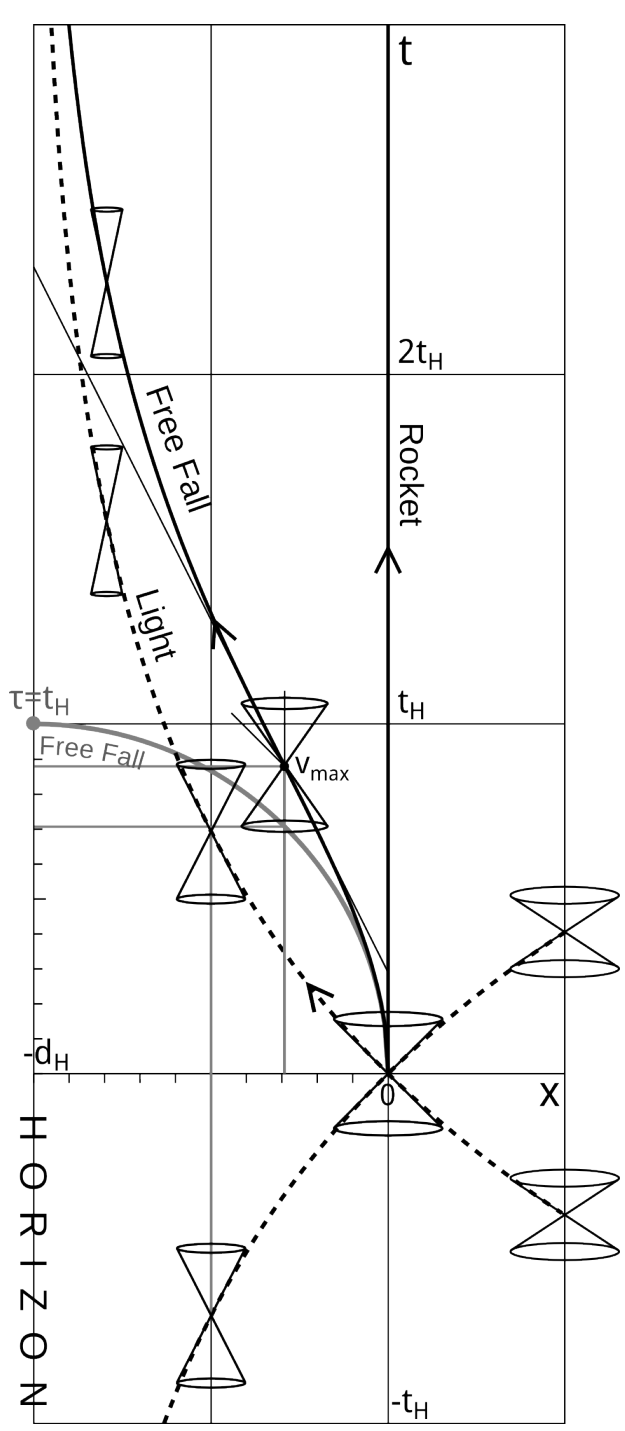

Figure 13: The Minkowski diagram in the non-inertial frame $(T, X)$. The cones indicate how the coordinate velocity of light varies with respect to $c$.

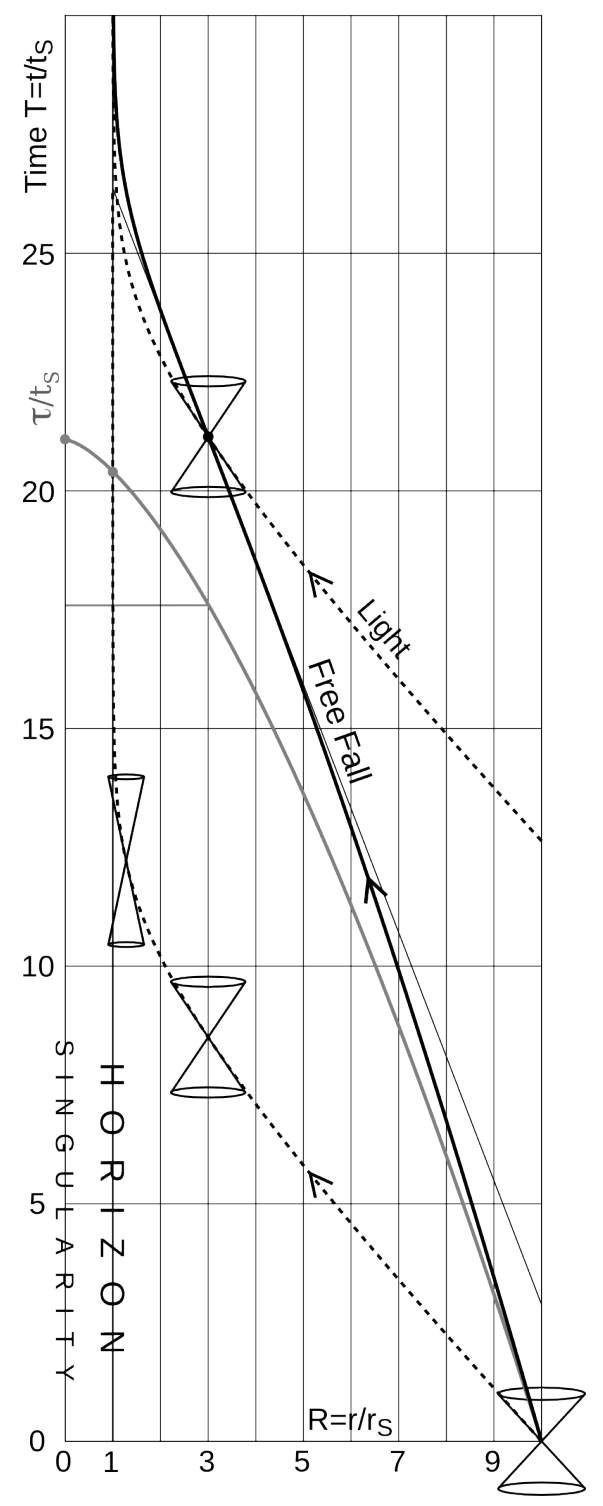

Figure 14: Minkowski diagram in the Schwarzschild frame $(T, R)$ [10] [11].

\subsection{Comparison with the black hole}

To broaden our view, we will look at another non-inertial frame of reference $R$ defined by the Schwarzschild metric. We have an observer at far distance from a spherical, static, and neutral star of mass $M$. There is also a horizon and the velocity and acceleration curves show analogies. On the other hand, space-time is curved; there is no global change of coordinates to an underlying inertial reference frame ; tidal effects will be present ; free fall is no longer adiabatic because of the emission of gravitational waves ; and, if the body is of non-negligible mass in front of the main star, the metric changes during the fall [12] [13] [14. We will use the Lagrangian approach to determine the equation of motion. The free-falling particle maximizes its proper time and follows a geodesic. We limit the study to the adiabatic radial fall of a test mass with a velocity of zero to infinity. The differential equation is then solved by a numerical approach for the plotting of the curves. 
Metric and Lagrangian for a particle:

$$
\begin{gathered}
d s^{2}=c^{2} d \tau^{2}=g(r) c^{2} d t^{2}-\frac{d r^{2}}{g(r)} \quad \text { with } \quad g=1-\frac{r_{S}}{r} \quad \text { and } \quad r_{S}=\frac{2 G M}{c^{2}}, \\
\tau=\int L(r, v) d t, \quad L=\sqrt{g-\frac{\beta^{2}}{g}} \quad \text { and } \quad L-\frac{\partial L}{\partial v} v=c s t .
\end{gathered}
$$

We obtain, with $R=r / r_{S}, T=t / t_{S}, t_{S}=r_{S} / c$ and $a_{S}=c^{2} / r_{S}$ :

$$
\beta=-\left(1-\frac{1}{R}\right) \frac{1}{\sqrt{R}} \quad \text { and } \quad A=\frac{d \beta}{d T}=\frac{\ddot{r}}{a_{S}}=\frac{1}{2 R^{2}}\left(1-\frac{1}{R}\right)\left(\frac{3}{R}-1\right) .
$$

We have $v_{\max }=2 /(3 \sqrt{3}) c \simeq 38 \% c$ at $r\left(v_{\max }\right)=3 r_{S}$ [15. Here too, the speed reaches a maximum and the acceleration changes of sign. The metric effects correspond to gravitation: they cannot be canceled over the whole space by a change of coordinates and are well real.

For Minkowskian observers: $d t_{\text {Mink }}=\sqrt{g} d t$ and $d r_{\text {Mink }}=d r / \sqrt{g}$ then $v_{\text {Mink }}\left(r_{v_{\max }}\right)=$ $c / \sqrt{3} \simeq 58 \% c$ and $v_{\max }=v_{\text {light }} / \sqrt{3}$ with $v_{\text {light }}\left(r_{v_{\max }}\right)=2 / 3 c$.

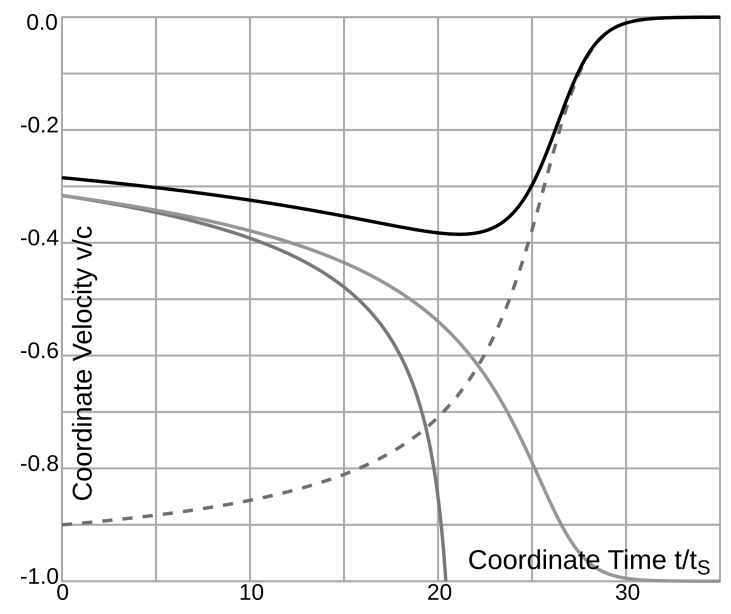

Figure 15: Falling velocity of a particle dropped without velocity from infinity. In dark gray, the curve according to Newton's laws. Dotted, the speed of light. In light gray, the speed for a local observer.

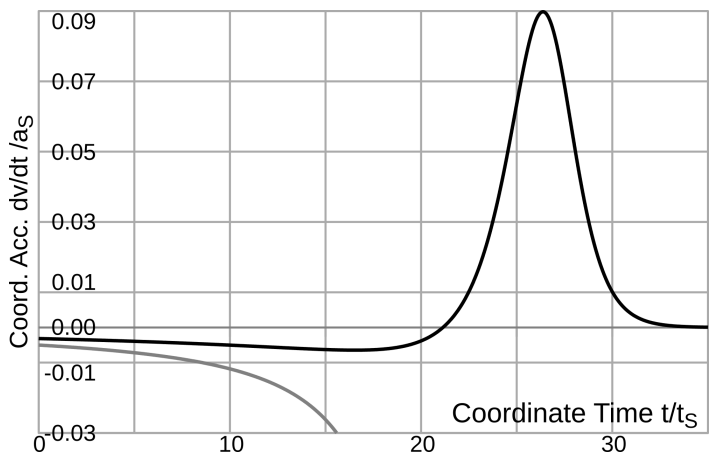

Figure 16: The acceleration of the particle in radial fall towards a black hole. In gray, the classical curve.

For the proper time the metric gives $\tau(R)=2 / 3\left(R_{0}^{3 / 2}-R^{3 / 2}\right) t_{S}$. For example, as shown in figure 14, with the initial condition $\tau=0$ when $T=0$ at $R_{0}=10$, when the speed of fall is maximum, $T \simeq 21$ and $\tau / t_{S} \simeq 18$. For an observer outside the black hole, the time of the falling object freezes on the horizon when the time of his clock tends towards infinity. Whereas for the observer in free fall, a finite proper time of about $2.8 t_{S}$ elapses between the maximum speed and the crossing of the horizon, and, nothing special happens during the crossing apart from the breaking of the causal link with the outside. Then $2 / 3 t_{S}$ of proper time is added until the singularity where the observer is destroyed. 


\section{$5 \quad$ Experimental aspects}

\subsection{Deviation measurement}

The difference is difficult to measure experimentally. We can have an accelerated rocket, but its width $d$ is very small in front of the horizon distance $d_{H}$, and, consequently, the observed deviation is tiny. The deviation depends on the ratio $D=d / d_{H}$. We perform a series expansions:

$$
\begin{aligned}
X=R_{X} \sqrt{1-\left(D-Y_{C}\right)^{2} / R_{Y}^{2}}-1 & \simeq \underbrace{\frac{D}{\tan \theta}}_{\text {rect.traj. }}-\underbrace{\frac{D^{2}}{2 \beta_{0}^{2} \sin ^{2} \theta}}_{\text {parabol. corr. }}+\underbrace{\frac{D^{3}}{2 \beta_{0}^{2} \sin ^{2} \theta \tan \theta}-\left[\frac{D^{4}}{8 \beta_{0}^{4}}\right]_{\theta= \pm \frac{\pi}{2}}+\ldots}_{\text {elliptical corrections }}
\end{aligned}
$$

The term in $D^{2}$ corresponds to the non-zero first order of the deviation due to the non-inertial character of the reference frame. The terms $D^{3}$ and beyond correspond to the deviation between classical theory and special relativity. For $d=10 \mathrm{~m}, a=10 \mathrm{~m} / \mathrm{s}^{2}$ and $\theta=45^{\circ}$, we have $|\Delta x| \simeq 11 \mathrm{fm}$ for the parabolic correction of a light ray, or, for the elliptic correction of a non-zero mass particle of velocity $v_{0}=10 \mathrm{~m} / \mathrm{s}$ (bell shot).

The deviation is extremely small and a direct measurement seems out of reach.

\subsection{Desynchronization of clocks}

On the other hand, with the precision of atomic clocks, the time deviation is experimentally accessible. For example, let say we launch a clock upwards with a vertical motion. Let us consider a maximum height reached of $h=45 \mathrm{~m}$. When the moving clock falls back in free fall on its return to the level of the clock that remained at $X=0$, with $a=10 \mathrm{~m} / \mathrm{s}^{2}$ and $v_{0} \simeq 30 \mathrm{~m} / \mathrm{s}$, the time difference between the two clocks is $\Delta t \simeq 10 \mathrm{fs}$ for a time of flight of $6 \mathrm{~s}$. For $\theta=0$ an expansion with respect to $\beta_{0}$ gives [8]:

$$
\begin{array}{r}
\Delta t / t_{H}=\tau / t_{H}-T=2\left(\gamma_{0} \beta_{0}-a t h \beta_{0}\right) \simeq \beta_{0}^{3} / 3 \\
\text { with } \quad H=\gamma_{0}-1 \simeq \beta_{0}^{2} / 2, \\
\text { and } \Delta t \simeq v_{0}^{3} /\left(3 a c^{2}\right) .
\end{array}
$$

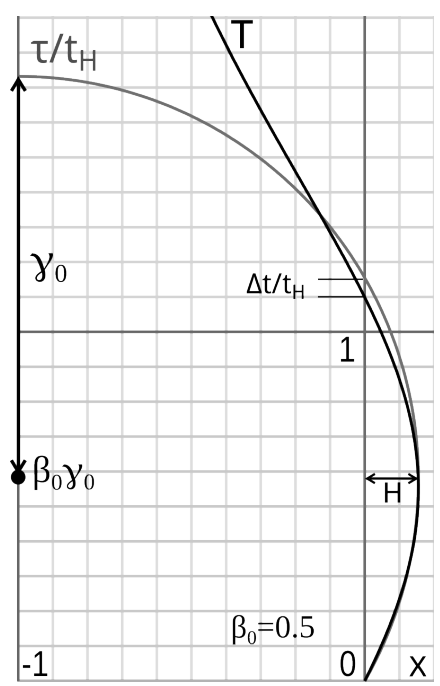

Figure 17: Minkowski diagram $(T, X)$ for a vertical launch. $\tau / t_{H}(X)$ draws a circle arc.

\subsection{Michelson interferometer}

We know the famous experiment of Michelson and Morley carried out from 1881 and that allowed to demonstrate the non-existence of the ether. The speed of light is therefore the same in all reference frames of inertia. What happens now if we perform the experiment in the accelerated reference frame?

In an inertial frame, or, here, horizontally in $R$, the rays take the same time to go and return on each of the arms and there is no optical path difference $\delta$ at $I$ (figure 18). We then turn the interferometer around the axis $\left(O M_{2}\right)$ so that arm 1 is up. As light travels faster than $c$ for positive $X$, the ray 1 that goes up and returns will be back before the ray 2: according to Eq.(4), for a round trip of ray $1, T=2 \ln (1+L) \simeq 2 L-L^{2}$. Ray 

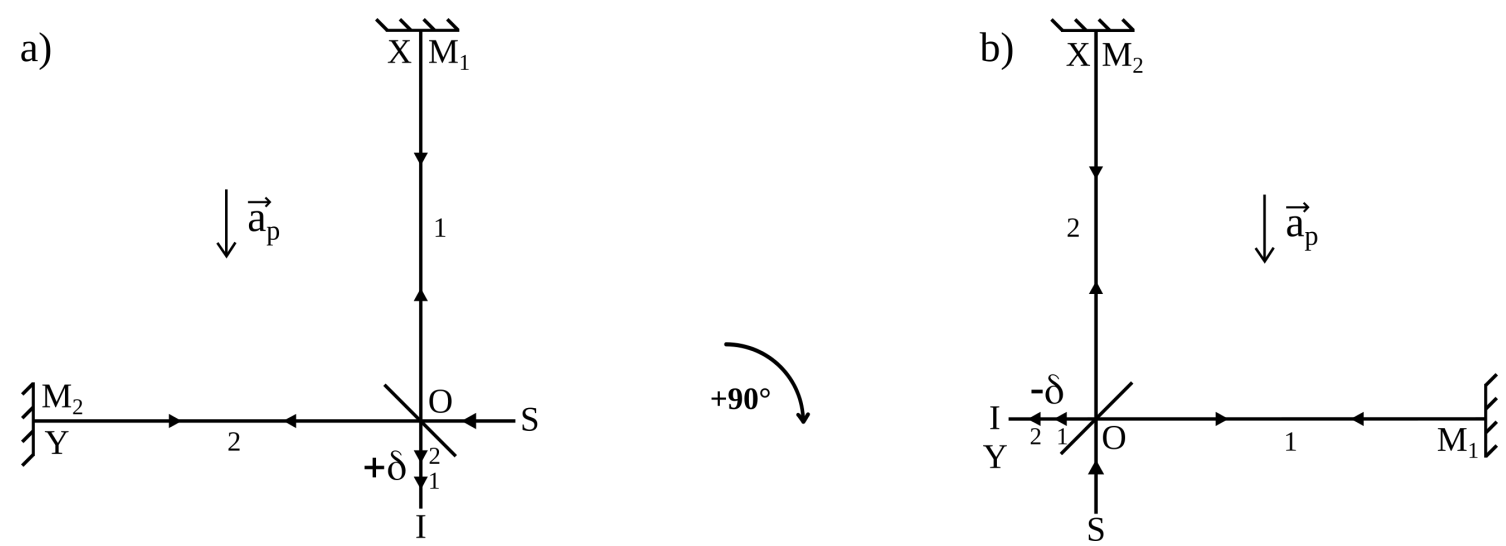

Figure 18: Michelson interferometer with two arms $O M_{1}$ and $O M_{2}$ of same length $l$.

2 follows arcs of circles, but this effect produces a smaller variation and we can consider the horizontal round trip to be along a straight line: according to Eq.(3), for a round trip of ray 2 , the lateral circular trajectory folds on $M_{2}$ and $T=\operatorname{ath}(2 L) \simeq 2 L+8 / 3 L^{3}$. We turn the interferometer again by $90^{\circ}$ but this time along the axis $(O z)$ as shown in figure 18. The situation is now the opposite and it is ray 2 that arrives first. We have drawn the world lines for configuration a) in figure 19. We deduce the value of the difference of pathlength and the number of fringes $n_{g}$ that scroll by tilting the interferometer from a) to b):

$$
n_{g}=\frac{2 \delta}{\lambda}=\frac{2}{\lambda}[\operatorname{ath}(2 L)-2 \ln (1+L)] d_{H} \simeq \frac{2 a l^{2}}{c^{2} \lambda} .
$$

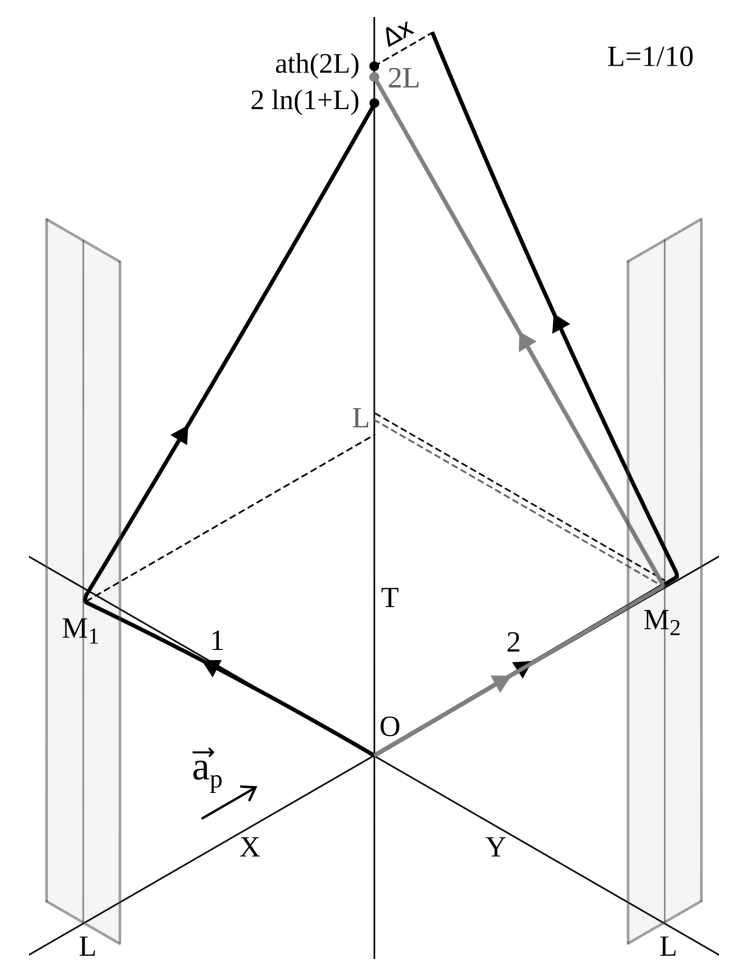

Figure 19: Minkowski diagram of the world lines of rays along the two arms of the interferometer for the case a).

For $a=10 \mathrm{~m} / \mathrm{s}^{2}, l=10 \mathrm{~m}$ and $\lambda=600 \mathrm{~nm}$, the calculation gives $n_{g} \simeq 0.4 \times 10^{-7}$. The variation is less than one ten millionth of a fringe. The larger $a$ and $l$ are, and the smaller $\lambda$ is, the more appreciable the effect will be. The spatial coherence of the interference is not lost with the vertical displacement of ray $2:|\Delta x| \simeq 22 \mathrm{fm}$. 
Also the arms are not perfectly rigid and we have to consider the mechanical constraints exerted on the vertical arm by the proper acceleration. We model the arm by a homogeneous cylinder of density $\rho$ and modulus of rigidity $E$. According to Hooke's law $\sigma=E \epsilon$ with $\sigma$ the pressure exerted and $\epsilon=\Delta l / l$ the relative deformation. The vertical arm becomes shorter and the ray will go up and return even faster. We calculate the compression $\Delta l$ by integrating over the entire arm, and deduce the difference in travel time induced by $a_{p}$ (acceleration assumed constant along the arm):

$$
\Delta l \simeq \frac{\rho a l^{2}}{2 E} \quad \text { then } \quad n_{a} \simeq \frac{4 \Delta l}{\lambda} \simeq \frac{2 \rho a l^{2}}{E \lambda} \quad \text { and } \quad \frac{n_{a}}{n_{g}} \simeq \frac{\rho}{E} c^{2} .
$$

Very rigid, low-density materials give an optical pathlength difference of the order of one fringe: $n_{a} \simeq 1$. The relative difference $n_{a} / n_{g}$ does not depend on $a, l$ or $\lambda$ but only on the mechanical properties of the material. The metric effect is tens of millions of times smaller than that due to mechanical constraints and will therefore be difficult to isolate experimentally. In a gravitational field, we will have the same results, because the spacetime curvature and tidal forces appear only at the following orders of the series expansions [8].

\subsection{Three rockets experiment}

We materialize an accelerated solid at the scale of a stellar system by synchronizing the parallel accelerations of three equidistant rockets.

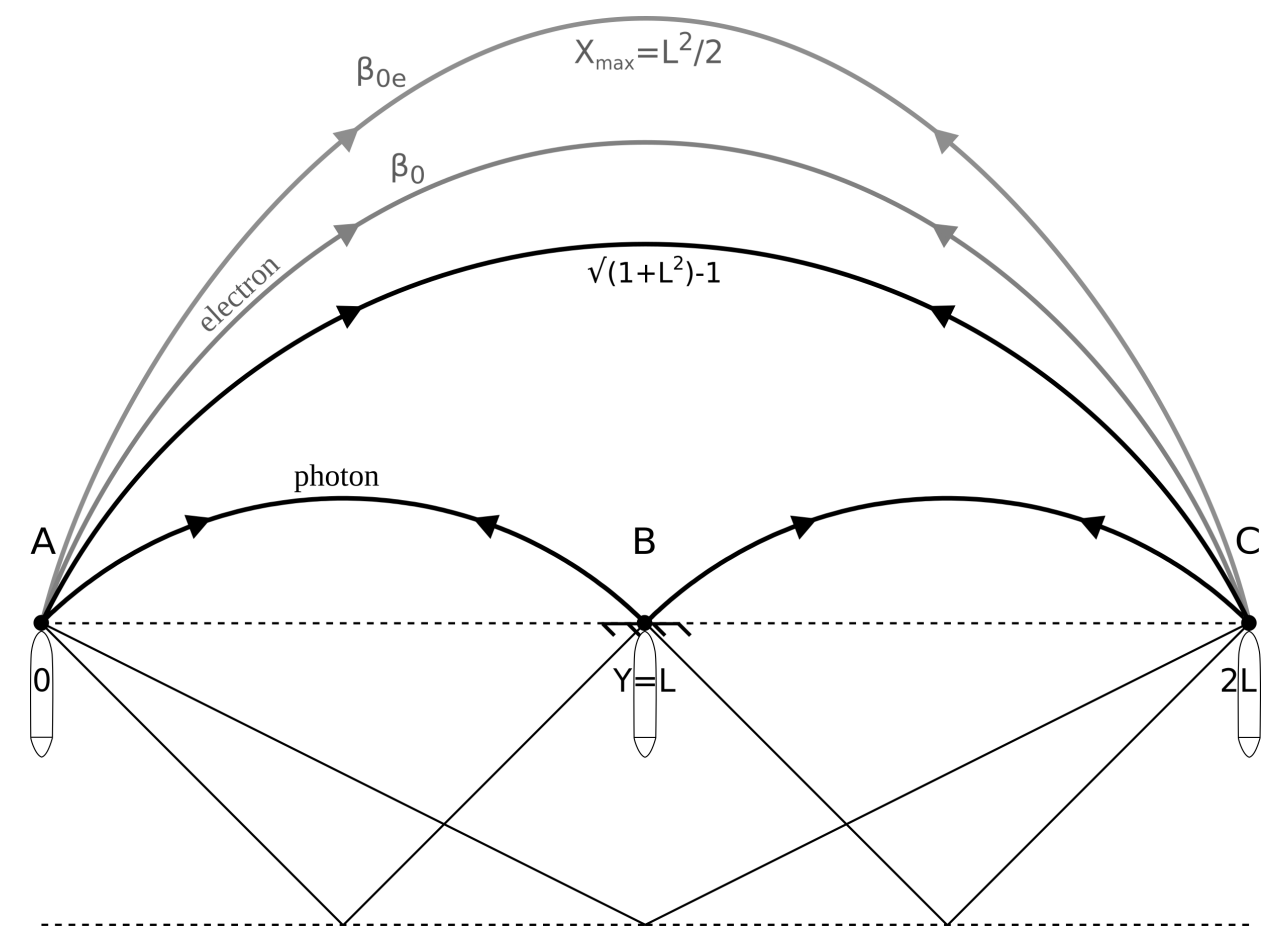

Figure 20: In black, light signals: $2 \Delta T=T_{A B}+T_{B C}>T_{A C}$. In gray, there is an initial velocity $\beta_{0 e}$ such that: $2 \Delta T=T_{A C}\left(\beta_{0 e}\right)$. Numerical values for $L=2: T_{A B}+T_{B C}=$ $2 \Delta T \simeq 3.53>T_{A C} \simeq 2.89, D_{A B}+D_{B C}=\sqrt{2} \pi<D_{A C} \simeq 1.58 \pi . \beta_{0 e}=\sqrt{17 / 18} \simeq 0.972$, $\gamma_{0 e}=3 \sqrt{2}, D_{A C}\left(\beta_{0 e}\right) \simeq 1.94 \pi$. 
The differences in distance and time for the two luminous paths $A B C B A$ and $A C A$ :

$$
\begin{gathered}
T_{A B C B A}-T_{A C A}=4 \times \operatorname{ach}\left(1+L^{2} / 2\right)-2 \times \operatorname{ach}\left(1+2 L^{2}\right) \simeq L^{3} / 2 \quad \text { if } \quad L<<1 \\
D_{A B C B A}-D_{A C A}=8 \sqrt{1+L^{2} / 4} \times \operatorname{atan} L / 2-4 \sqrt{1+L^{2}} \times \operatorname{atan} L \simeq-L^{3} / 2
\end{gathered}
$$

For three rockets $A, B$ and $C$, one astronomical unit apart and with the accelerations $a=10 \mathrm{~m} / \mathrm{s}^{2}, t_{A B C B A}-t_{A C A} \simeq 69 \mathrm{~ns}$.

If we assume that, as in an inertial reference frame, the speed of light is constant, we obtain a paradox: the photon that runs the longest distance returns first. Perhaps, even more paradoxical here, a particle of matter, with an initial speed $\beta_{0}$ between 1 and $\beta_{0 e}$, will also arrive before light, while covering a greater distance and going, a priori, less quickly. We find a relativistic version of the tortoise and the hare paradox of La Fontaine's Fables.

For calculations:

$$
\begin{aligned}
& T_{A B C B A}-T_{A C A}\left(\beta_{0}\right)=4 \times \operatorname{ach}\left(1+L^{2} / 2\right)-2 \times \operatorname{ach}\left[\frac{1-\sqrt{1-\left(1-\beta_{0}{ }^{2}\right)\left(1+\beta_{0}{ }^{2}+4 L^{2}\right)}}{1-\beta_{0}{ }^{2}}\right] \\
& \simeq L^{3} / 2-4 L\left(1-\beta_{0}\right) \quad \text { if } \quad L<<1 \quad \text { and } \quad \beta_{0} \simeq 1 \\
& \beta_{0 e}=\sqrt{\frac{L^{2}\left(1+L^{2} / 4\right)^{2}+1}{\left(1+L^{2} / 2\right)^{2}\left(1+L^{2} / 4\right)}} \simeq 1-L^{2} / 8 \\
& \text { and } \gamma_{0 e}=\frac{2+L^{2}}{L} \sqrt{1+L^{2} / 4} \simeq 2 / L \quad \text { for } \quad L<<1
\end{aligned}
$$

For an electron with $\gamma_{0}=\sqrt{2} \gamma_{0 e}$, again for $a=10 \mathrm{~m} / \mathrm{s}^{2}$ and $l=1 a u, L=1 / 60000$ and, as $1-\beta \simeq 1 / 2 \gamma^{2}, t_{A B C B A}-t_{A C A}\left(\beta_{0}\right) \simeq 35 n s$.

At the start of the race the fast photon is ahead of the massive electron. The electron then takes a trajectory of a single jump, which is longer, but which brings the electron higher where time goes faster. Seen from above the time of the photon freezes and so the electron manages to overtake the opponent. Already in $C$, the electron bounces before the photon.

\section{Conclusion}

The calculations remain relatively simple and allow students to become familiar with concepts that are partly reused in general relativity. At the same time, this textbook case helps to avoid a lot of confusion in relativity. Pushing special relativity to its last non-inertial limits, where an underlying global Minkowskian framework persists, makes it easy to implement a progressive learning pedagogy. Moreover, the theoretical vision is broadened, and perhaps one day direct experimental verifications will be feasible.

\section{References}

[1] A. Einstein, Relativity: The Special and the General Theory, in chapter: A few inferences from the general theory of relativity (1917). 
[2] C. Møller, The Theory of Relativity, See p253 in Oxford 1st Ed. (1952).

[3] E. A. Desloge and R. J. Philpott, Uniformly accelerated reference frames in special relativity, Am. J. Phys. 55(3), 252 (1987), doi:10.1119/1.15197.

[4] L. Landau and E. Lifchitz, The classical Theory of Fields, § Distances and time intervals $\S$ The synchronous reference system.

[5] E. A. Desloge, Spatial geometry in a uniformly accelerating reference frame, Am. J. Phys. 57(7), 598 (1989), doi:10.1119/1.15953.

[6] W. Rindler, Relativity: Special, General, and Cosmological, Oxford Univ. Press, See p71 and 282 (Exercise 12.7) $2^{\text {nd }}$ Ed. (2006).

[7] C. Semay, Observer with a constant proper acceleration, Eur. J. Phys. 27(5), 1157 (2006), doi:10.1088/0143-0807/27/5/015

[8] M. Rouaud, Special Relativity, A Geometric Approach, ISBN 9782954930930, See p243, 167, 387 and 157 (2020), http://www.voyagepourproxima.fr/SR.pdf.

[9] J. D. Hamilton, The uniformly accelerated reference frame, Am. J. Phys. 46(1), 83 (1978), doi:10.1119/1.11169.

[10] K. S. Thorne, C. W. Misner and J. A. Wheeler, Gravitation, Freeman, ISBN 0716703440, See p848 (1971).

[11] P. Tourrenc, Relativity and Gravitation, Cambridge University Press (1997).

[12] A. D. Spallicci and P. Ritter, A fully relativistic radial fall, Int. J. Geom. Methods Mod. Phys. 11(10) (2014), doi:10.1142/S021988781450090X.

[13] E. A. Desloge, Nonequivalence of a uniformly accelerating reference frame and a frame at rest in a uniform gravitational field, Am. J. Phys. 57(12), 1121 (1989), doi: $10.1119 / 1.15802$

[14] G. Muñoz and P. Jones, The equivalence principle, uniformly accelerated reference frames, and the uniform gravitational field, Am. J. Phys. 78(4), 377 (2010), doi: $10.1119 / 1.3272719$.

[15] S. I. Blinnikov, L. B. Okun' and M. I. Vysotskii, Critical velocities $c / \sqrt{3}$ and $c / \sqrt{2}$ in the general theory of relativity, Physics-Uspekhi 46(10), 1099 (2003), doi:10.1070/pu2003v046n10abeh001661. 